\title{
Cu/MWCNT Interfaces in Room Temperature Aged Nanocomposites Revealed by HRTEM and EELS
}

\author{
M.E. Mendoza ${ }^{1}$, I.G. Solórzano ${ }^{2}$, T. Aoki ${ }^{3}$, D.J. Smith ${ }^{3}$ \\ ${ }^{1}$ Materials Division, Inmetro, Duque de Caxias, Rio de Janeiro, 25250-020, Brazil. \\ 2 Department of Materials Engineering, PUC-Rio, Rio de Janeiro, PO Box 238097, Brazil \\ ${ }^{3}$ Department of Physics, Arizona State University, Tempe, AZ 85287-1504, USA
}

The success of a metal-matrix bulk nanocomposite depends upon the nature, volume fraction, distribution of reinforcement, and the interface with the matrix. Multi-walled carbon nanotubes (MWCNT) have significantly improved the mechanical properties of composites when reinforced. However, transport properties (namely electric) have been observed to decrease [1,2]. Little information about the Cu-CNT interface structure and chemistry (responsible for the mechanical and transport properties) has been reported, mainly due to the lower solubility of $\mathrm{C}$ in $\mathrm{Cu}$. In this study, Cu-MWCNT bulk nanocomposites were produced by chemical synthesis followed by thermo mechanical processing using spark plasma sintering (SPS).

This present investigation reports the structural and spectroscopic characterization of a $\mathrm{Cu}-0.5 \mathrm{wt}$ (\%) and Cu-5 wt (\%) MWCNT nanocomposites using TEM and EELS. Nanocomposite powders were synthesized according to the procedure described elsewhere [3]. Bulk pellets were produced by sintering the powder material using a Dr. Sinter lab SPS 1050 at $600^{\circ} \mathrm{C}$ by $5 \mathrm{~min}$, heating rate of 100 ${ }^{\circ} \mathrm{C} / \mathrm{min}, 70 \mathrm{MPa}$ compression under $10^{-3}$ Torr. A JEOL $4000 \mathrm{EX}$ operating at $400 \mathrm{kV}$ and a JEOL ARM200F operating at 200KV were used for characterization. Sample preparation was done using a Nanonova focused-ion-beam instrument. After consolidation into pellets and sintering, the Cu matrix presented a good consolidation state, exhibiting equiaxed grains with characteristic annealing twins and also some heterogeneous grain growth, with grain size in the $50 \mathrm{~nm}-3 \mu \mathrm{m}$ range, as shown in Fig.1a. An important finding is the transformation of most MWCNTS into graphite and also amorphous carbon, mainly at copper grain boundaries. It is believed that high current density, as well as high temperature and pressure during processing, played a major role to catalyze this transformation. However, some MWCNTs with diameters above $50 \mathrm{~nm}$ were found (inset Fig. 1a). Fourier analysis has shown localized regions (5-20 nm length) at the Cu-MWCNT interface where inter-diffusion between $\mathrm{Cu}$ and $\mathrm{C}$ appears to have taken place, as observed in the HRTEM image of Fig. 1b. This finding agrees with simulations reported by Dorfman et al [4]. Note that although the sample was analyzed at $400 \mathrm{kV}$, it did not suffer detectable structural changes and offered good stability during TEM operations. EELS line scan across the interface confirmed the presence of inter-diffusion region containing $\mathrm{C}, \mathrm{Cu}$ and $\mathrm{O}$, as shown in Fig 2. A 10-nm layer of $\mathrm{Cu}_{2} \mathrm{O}$ between the MWCNT and $\mathrm{Cu}$ matrix suggests an oxidation process suffered by the thin foil at room temperature over a period of about 30 months. In addition, such long aging at RT has allowed $\mathrm{Cu}_{2} \mathrm{O}$ precipitation in the copper matrix (Fig. 3).

References

[1] K. T. Kim, et al. Materials Science and Engineering A 430 ( 2006) 27-33.

[2] K.Tae, et al. Materials Science and Engineering A 449-451 (2007) 46-50.

[3] M.E. Mendoza, I.G. Solorzano, E.A. Brocchi. Materials Science and Engineering A 544(2012)21-26.

[4] S. Dorfman et al. Materials Science and Engineering C, (2001) 191-193.

[5] The authors acknowledge NSF (USA), AFOSR (USA) and CNPq (Brazil) for financial support. 

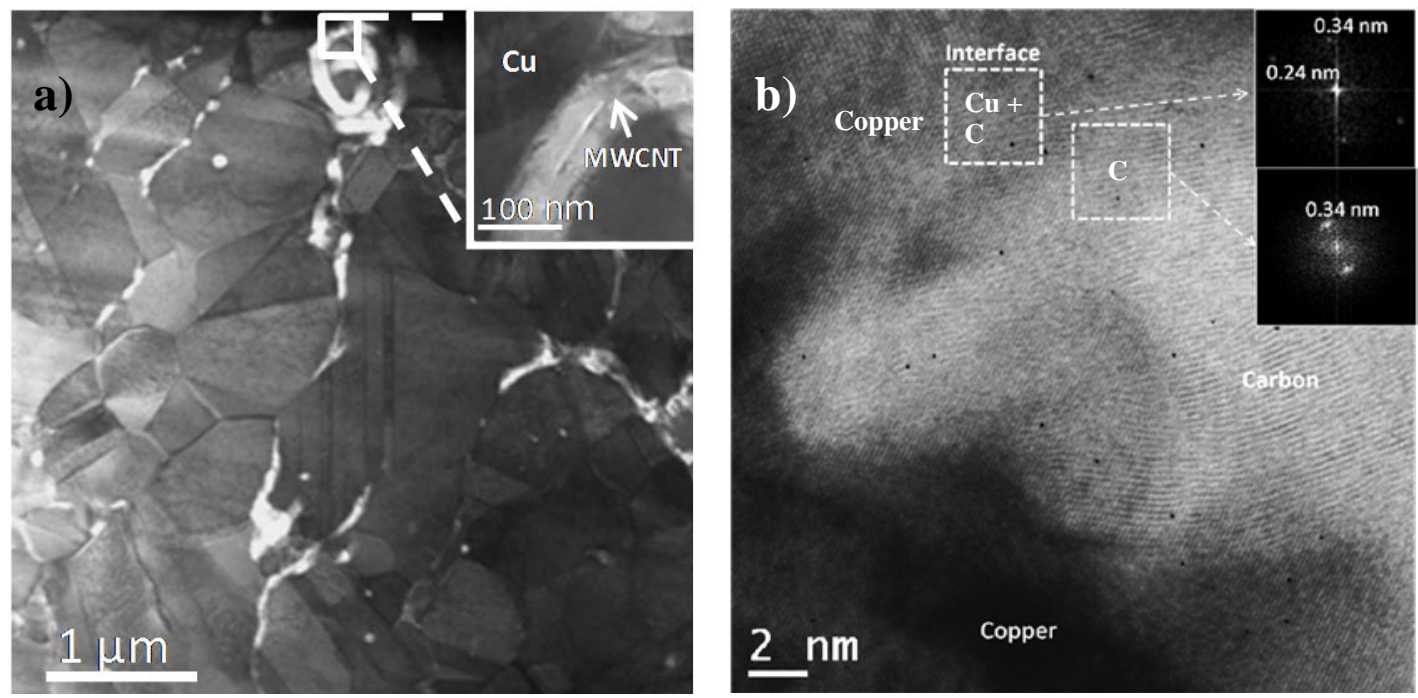

Fig. 1a) BF-STEM image of Cu-0.5 wt\% MWCNT showing significant $\mathrm{C}$ segregation at Cu grain boundaries. Fig $1 \mathrm{~b}$ ) HRTEM image at the $\mathrm{Cu} /$ transformed MWCNT interface.

a)

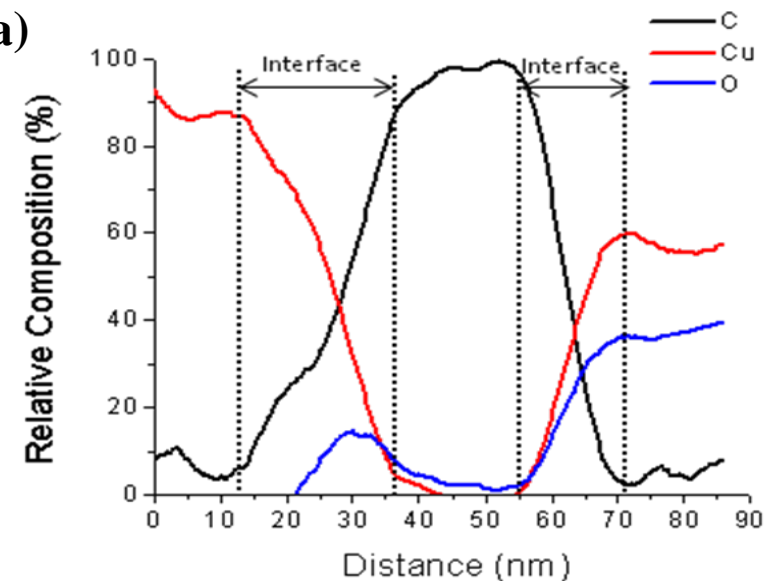

b)

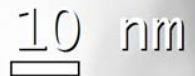

Fig. 2a. Cu-5 wt\% MWCNT: relative composition of C, $\mathrm{Cu}$ and $\mathrm{O}$ at the interface region measured by EELS. b) ADF STEM of the measured region.

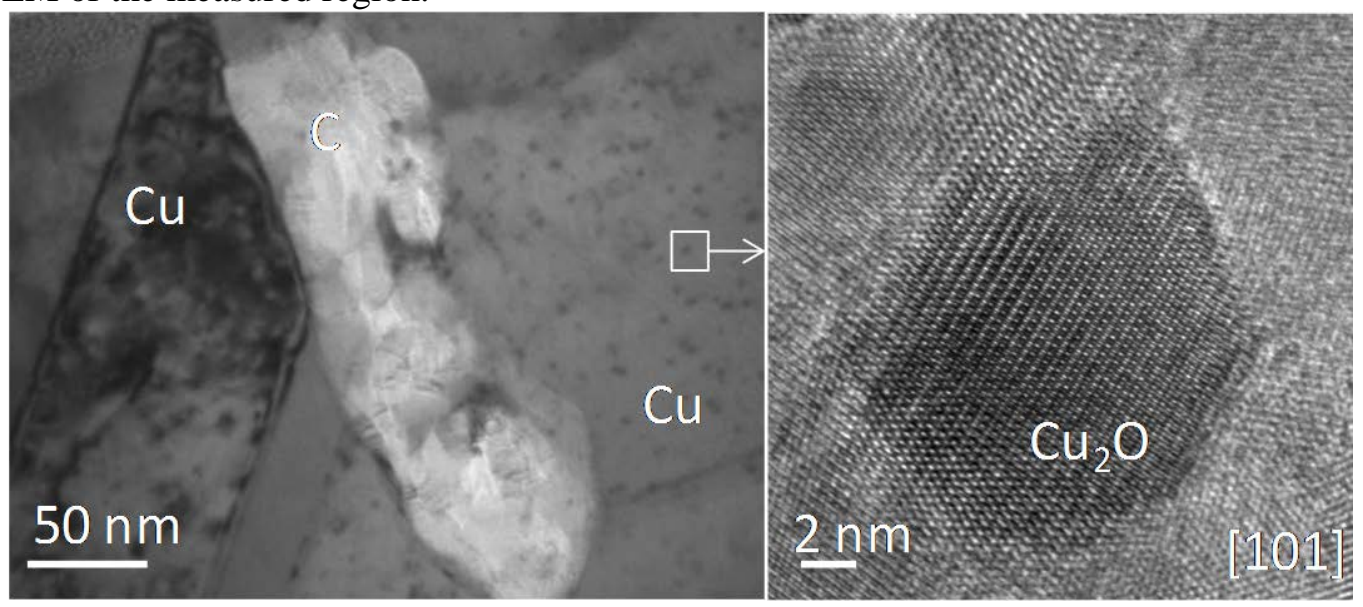

Fig. 3a. Bright-field TEM image of $\mathrm{Cu}$ matrix exhibiting extensive agglomeration and $\mathrm{Cu}_{2} \mathrm{O}$ precipitates. b) HRTEM of $\mathrm{Cu}_{2} \mathrm{O}$ precipitate. 